\title{
DEVELOPMENT AND APPLICATION OF ADVANCED ONE-POINT TURBULENCE MODELS
}

\author{
Charles G. Speziale
}

January 1996

Technical Report No. AM- 96-002

\section{DISCLAIMER}

\begin{abstract}
This report was prepared as an account of work sponsored by an agency of the United States Government. Neither the United States Government nor any agency thereof, nor any of their employees, makes any warranty, express or implied, or assumes any legal liability or responsibility for the accuracy, completeness, or usefulness of any information, apparatus, product, or process disclosed, or represents that its use would not infringe privately owned rights. Reference herein to any specific commercial product, process, or service by trade name, trademark, manufacturer, or otherwise does not necessarily constitute or imply its endorsement, recommendation, or favoring by the United States Government or any agency thereof. The views and opinions of authors expressed herein do not necessarily state or reflect those of the United States Government or any agency thereof.
\end{abstract}

Final Technical Report:

Los Alamos National Laboratory

Contract No. $326740015-3 \mathrm{C}$ 


\section{DISCLAIMER}

Portions of this document may be illegible in electronic image products. Images are produced from the best available original document. 


\title{
DEVELOPMENT AND APPLICATION OF ADVANCED ONE-POINT TURBULENCE MODELS*
}

\author{
Charles G. Speziale \\ Aerospace \& Mechanical Engineering Department \\ Boston University \\ Boston, MA 02215
}

\begin{abstract}
Full self-preserving solutions in isotropic decay and homogeneous shear flow turbulence have been examined from a basic theoretical standpoint. These constitute solutions for the two-point double and triple velocity correlations that are self-similar at all scales. Consistent with earlier studies, it was found that both isotropic decay and homogeneous shear flow turbulence have full self-preserving solutions. At high Reynolds numbers - with finite viscosity - the full self-preserving solution for isotropic decay corresponds to a $t^{-1}$ power-law decay whereas that for homogeneous shear flow corresponds to a production-equals-dissipation equilibrium. An alternative derivation of the isotropic results based on group theory considerations was recently achieved by T. Clark and C. Zemach of Los Alamos. These results suggest that such self-preserving solutions are associated with a singularity in the energy spectrum tensor (i.e., the Fourier transform of the two-point double velocity correlation tensor) at zero wave vector. This can have a profound effect on turbulence models. The ultimate goal is to use these two-point results for the development of improved one-point turbulence models for the solution of practical turbulent flows of scientific and engineering interest.
\end{abstract}

${ }^{*}$ Research sponsored by Los Alamos Contract No. 326740015-3C. 


\section{INTRODUCTION}

The main overall goal of this research effort was to enlist the results of two-point closures for the ultimate development of improved one-point closures for the prediction of turbulent flows of scientific and engineering importance. This was a collaborative effort with T. Clark and C. Zemach of Los Alamos National Laboratory and much of the research was accomplished during period visits to Los Alamos that were budgeted for. During the past year, it was decided to concentrate on the question of full self-preserving solutions in homogeneous turbulence, with particular attention paid to the benchmark cases of isotropic decay and homogeneous shear flow. These constitute benchmark cases that have been analyzed both with one-point and two-point closures over the years. The fundamental questions addressed ultimately have important implications for the modeling of the turbulent dissipation rate which plays a pivotal role in one-point closures. It also can shed new light on the origins of a $t^{-1}$ power law decay in isotropic turbulence and a production-equals-dissipation equilibrium in homogeneous shear flow. Such fundamental issues themselves - while they have important implications for both the modeling and numerical simulation of practical turbulent flows are crucial for understanding the basic physics of turbulence.

\section{RESEARCH ACCOMPLISHED}

The main focus of this research effort for the past year concentrated on self-preserving homogeneous turbulent flows as mentioned above. Homogeneous turbulent flows are fully self-preserving if all relevant two-point correlations - most notably, including the two-point double velocity correlation tensor - are self-similar at all scales. Here, self-similarity is established by a single governing length scale. New results were obtained concerning full self-preserving homogeneous turbulent shear flow. In particular, it was found that:

(1) With non-zero viscosity, the only full self-preserving solution for homogeneous turbulent shear flow is a production-equals-dissipation equilibrium $(\mathcal{P}=\varepsilon)$ where the turbulent kinetic energy and dissipation rate saturate to finite equilibrium values. Here, the Taylor microscale $\lambda$ is the governing length scale that establishes self-similarity. This type of production-equals-dissipation equilibrium in homogeneous shear flow had been hypothesized earlier by Bernard and Speziale (1992) based on different arguments.

(2) In the absence of viscosity, there is a self-preserving solution where the turbulent 
kinetic energy and dissipation rate grow exponentially at the same rate. An equilibrium state is ultimately achieved by their ratio - the turbulent time scale. Here, the relevant governing length scale that establishes self-similarity is the turbulent macroscale $K^{3 / 2} / \varepsilon$. This is formally, a partial self-preserving solution since viscosity is neglected.

To achieve this production-equals-dissipation equilibrium discussed in point (1) - with bounded turbulent kinetic energy and dissipation - it appears that the initial conditions, which are taken to be isotropic, must also be fully self-preserving. Preliminary computations with the two-point closure of Clark and Zemach (1995), shown in Figure 1, tend to support this (the full self-preserving case corresponds to $n=1$ ). When starting from a full self-preserving isotropic decay, the flow attempts to approach a production-equals-dissipation equilibrium which is self-similar at all scales - when a uniform shear is applied (see Figure 1). There are, however, some questions that need to be resolved in regard to the occurrence of singularities in this solution. For other initial conditions, the turbulent kinetic energy and dissipation rate grow exponentially as shown in Figure 1. The mathematical details of these full selfpreserving results for homogeneous shear flow discussed in points (1) - (2) are provided in the Appendix.

Results concerning full self-preservation in isotropic turbulence were further clarified. Speziale and Bernard (1992) had earlier shown that, at high-Reynolds-numbers, the only full self-preserving solution corresponds to a $t^{-1}$ power law decay. Here, it was shown that this solution corresponds to an energy spectrum tensor that behaves like $k^{-1}$ near zero wavenumbers - a result obtained earlier by Clark and Zemach based on group theoretical considerations. This suggests an isolated singularity at zero wavenumbers and the intriguing possibility that a $t^{-1}$ power law decay in isotropic turbulence may emanate from a finite time singularity of the Fourier transform of the Navier-Stokes equations. The mathematical details of the derivation of this result is also provided in the Appendix.

\section{FUTURE RESEARCH}

Future research will concentrate on the role of the initial conditions in achieving selfpreservation. In addition, the intriguing possibility of a finite time singularity of the NavierStokes equations in Fourier space will be explored in more depth as a possible avenue to full self-preservation and a $t^{-1}$ power law decay in isotropic turbulence. In this regard, several 
features regarding the two-point closure of Clark and Zemach (1995) need to be clarified and further improved upon. These results can have a profound effect on one-point turbulence models for practical turbulent flows. Future research will attempt to focus more on this issue. 


\section{APPENDIX}

Our analysis of self-preservation is based on the Navier-Stokes and continuity equations given by:

$$
\begin{gathered}
\frac{\partial v_{i}}{\partial t}+v_{j} \frac{\partial v_{i}}{\partial x_{j}}=-\frac{\partial P}{\partial x_{i}}+\nu \nabla^{2} v_{i} \\
\frac{\partial v_{i}}{\partial x_{i}}=0
\end{gathered}
$$

where $v_{i}$ is the velocity vector, $P$ is the kinematic pressure and $\nu$ is the kinematic viscosity. For homogeneous turbulent flows, the continuity equation (2) can be used to project the pressure out of the problem. The velocity and pressure are decomposed into ensemble mean and fluctuating parts, respectively, as follows:

$$
v_{i}=\bar{v}_{i}+u_{i}, \quad P=\bar{P}+p
$$

From the fluctuating velocity $u_{i}$ in (3), we can construct the two-point double velocity correlation which, for homogeneous turbulent flows, can be written as

$$
R_{i j}(\mathbf{r}, t)=\overline{u_{i}(\mathbf{x}, t) u_{j}(\mathbf{x}+\mathbf{r}, t)}
$$

The Fourier transform of (4) is referred to as the energy spectrum tensor, $R_{i j}(\mathbf{k}, t)$, where $\mathbf{k}$ is the wavevector. In homogeneous turbulence, the fluctuating velocity is a solution of the transport equation

$$
\frac{\partial u_{i}}{\partial t}+\bar{v}_{j} \frac{\partial u_{i}}{\partial x_{j}}=-u_{j} \frac{\partial u_{i}}{\partial x_{j}}-u_{j} \frac{\partial \bar{v}_{i}}{\partial x_{j}}-\frac{\partial p}{\partial x_{i}}+\nu \nabla^{2} u_{i}
$$

which is obtained by subtracting (1) from its ensemble mean. After projecting the pressure out, a second (two-point) moment of (5) yields a transport equation for the two-point double velocity correlation tensor $R_{i j}(\mathbf{r}, t)$. By taking the Fourier transform of this equation we obtain the transport equation for the energy spectrum tensor which is given by:

$$
\begin{aligned}
\frac{\partial R_{i j}}{\partial t}= & -2 \nu k^{2} R_{i j}+G_{m n}\left[-\delta_{i m} R_{n j}-\delta_{j m} R_{i n}+2 \frac{k_{m} k_{i}}{k^{2}} R_{n j}\right. \\
& \left.+\frac{2 k_{m} k_{j}}{k^{2}} R_{i n}+\frac{\partial}{\partial k_{n}}\left(k_{m} R_{i j}\right)\right]+T_{i j}
\end{aligned}
$$

where $G_{i j} \equiv \partial \bar{v}_{i} / \partial x_{j}$ and $T_{i j}$ is the transfer (this is related to the Fourier transform of the two-point triple velocity correlation tensor; cf. Clark and Zemach 1995). For homogeneous shear flow,

$$
G_{i j}=S \delta_{i 1} \delta_{j 2}
$$


where $S$ is the shear rate which is constant. This leads to the transport equation

$$
\frac{\partial R_{i j}}{\partial t}=-2 \nu k^{2} R_{i j}+S\left[-\delta_{i 1} R_{2 j}-\delta_{j 1} R_{i 2}+2 \frac{k_{1} k_{i}}{k^{2}} R_{2 j}+2 \frac{k_{1} k_{j}}{k^{2}} R_{i 2}+k_{1} \frac{\partial R_{i j}}{\partial k_{2}}\right]+T_{i j} .
$$

For full self-preservation, we must have

$$
R_{i j}(\mathbf{k}, t)=K L^{3} R_{i j}^{*}\left(\mathbf{k}^{*}\right)
$$

where

$$
\mathbf{k}^{*}=\mathbf{k} L
$$

given that $K$ is the turbulent kinetic energy and $L=L(t)$ is a length scale that establishes similarity. We must also have

$$
T_{i j}(\mathbf{k}, t)=K^{3 / 2} L^{2} T_{i j}^{*}\left(\mathbf{k}^{*}\right)
$$

for full self-preservation. Hence, it follows that the substitution of $(9)-(10)$ into (8) yields:

$$
\begin{aligned}
\frac{\partial R_{i j}}{\partial t} \equiv & \left(\dot{K} L^{3}+3 K L^{2} \dot{L}\right) R_{i j}^{*}+K L^{2} \dot{L} k_{m}^{*} \frac{\partial R_{i j}^{*}}{\partial k_{m}^{*}} \\
= & -2 \nu K L k^{* 2} R_{i j}^{*}+S K L^{3}\left[-\delta_{i 1} R_{2 j}^{*}\right. \\
& -\delta_{j 1} R_{i 2}^{*}+2 \frac{k_{1}^{*} k_{i}^{*}}{k^{* 2}} R_{2 j}^{*}+2 \frac{k_{1}^{*} k_{j}^{*}}{k^{* 2}} R_{i 2}^{*} \\
& \left.+k_{1}^{*} \frac{\partial R_{i j}^{*}}{\partial k_{2}^{*}}\right]+K^{3 / 2} L^{2} T_{i j}^{*} .
\end{aligned}
$$

Dividing by $\dot{K} L^{3}$, it follows that we must then have the following constraints satisfied for full self-preservation:

$$
\begin{aligned}
\frac{K}{\dot{K}} \frac{\dot{L}}{L} & =\text { constant } \\
\nu \frac{K}{\dot{K}} \frac{1}{L^{2}} & =\text { constant } \\
S \frac{K}{\dot{K}} & =\text { constant } \\
\frac{K^{3 / 2}}{\dot{K} L} & =\text { constant. }
\end{aligned}
$$

For the limiting case of isotropic turbulence $(S=0)$, Eqs. (12)-(15) reduce to:

$$
\frac{K}{\varepsilon} \frac{\dot{L}}{L}=\text { constant }
$$




$$
\begin{aligned}
& \frac{\nu K}{\varepsilon} \frac{1}{L^{2}}=\text { constant } \\
& \frac{K^{3 / 2}}{\varepsilon L}=\text { constant }
\end{aligned}
$$

where $\varepsilon$ is the turbulent dissipation rate. In deriving (16) - (18) we have made use of the fact that

$$
\dot{K}=-\varepsilon
$$

in isotropic turbulence. These equations imply that the turbulence Reynolds number $R_{t}=$ $K^{2} / \nu \varepsilon$ is a constant - denoted by $R_{t \infty}$ - which corresponds to a $K \sim t^{-1}$ power law decay (see Bernard and Speziale 1992). If $R_{t_{\infty}}>0$, we have

$$
L \propto \frac{K^{3 / 2}}{\varepsilon} \propto \lambda
$$

where

$$
\lambda=\sqrt{10 \nu K / \varepsilon} \quad(\text { Taylor microscale) }
$$

If, on the other hand, $R_{t_{\infty}}=0$, then $L \propto \lambda$ and we have the associated power law decay, $K \sim t^{-\alpha}$ where the exponent $\alpha$ depends on the initial conditions. Gaussianity yields $\alpha=2.5$ which corresponds to the final period of decay.

If $\nu=0$ :

$$
\begin{gathered}
L \propto \frac{K^{3 / 2}}{\varepsilon} \\
K \sim t^{-\alpha}
\end{gathered}
$$

which is a partial self-preserving solution where we typically have $1<\alpha<1.42$.

For Homogeneous Shear Flow $(S>0)$ we must have (see Bernard and Speziale 1992):

$$
\begin{gathered}
K \sim e^{\Lambda t} \\
\varepsilon \sim e^{\Lambda t}
\end{gathered}
$$

where there is a production-equals-dissipation equilibrium in the limit of $\Lambda=0$.

Then, Eq. (13), which states that

$$
\frac{\nu K}{\dot{K}} \frac{1}{L^{2}}=\text { constant }
$$

implies that

$$
L \sim \text { constant }
$$


This causes $K^{3 / 2} / \dot{K} \rightarrow \infty$ as $t \rightarrow \infty$, which renders a zero transfer, i.e.,

$$
T_{i j}^{*} \rightarrow 0
$$

when $\Lambda>0$. This is clearly an unphysical solution (one cannot have an isotropic decay, at high Reynolds numbers, with zero transfer).

Hence, we must have $\Lambda=0$. Then, it follows that $\dot{K}=0$ and (12) $-(15)$ implies that

$$
\frac{\dot{L}}{L}=0
$$

and hence that

$$
L=\text { constant }
$$

In homogeneous turbulent flows, the Navier-Stokes equations imply that

$$
\dot{K}=\mathcal{P}-\varepsilon
$$

where $\mathcal{P}$ is the turbulence production. Since, $\dot{K}=0$, we must thus have a production-equalsdissipation equilibrium

$$
\mathcal{P}=\varepsilon
$$

for full self-preservation, if $\nu>0$.

If $\nu=0$, then, Eqs. (12), (14) and (15) imply that

$$
\begin{aligned}
& K \sim e^{\Lambda t}, \varepsilon \sim e^{\Lambda t}(\Lambda>0) \\
& L \propto \frac{K^{3 / 2}}{\dot{K}} \propto \frac{K^{3 / 2}}{\varepsilon} .
\end{aligned}
$$

This is formally a partial self-preserving solution.

\section{Isotropic Decay}

For $k^{*} \ll 1$, we have

$$
R_{i i}^{*} \sim k^{* n} \equiv \beta k^{* n}
$$

(see Clark and Zemach 1995). Then, it follows from (11) that:

$$
\left(\dot{K} L^{3}+3 K L^{2} \dot{L}\right) \beta k^{* n}+K L^{2} \dot{L}\left(\beta n k^{* n}\right)=0 .
$$

if $T_{i i}^{*}\left(k^{*}\right) \sim k^{* m},(m>n)$. We must then have:

$$
\dot{K} L^{3}+3 K L^{2} \dot{L}+n K L^{2} \dot{L}=0 .
$$


Then, it follows that

$$
\dot{K} L^{2}+(3+n) K L \dot{L}=0
$$

or, equivalently, that

$$
\overline{\overline{K L^{2}}}+(n+1) K L \dot{L}=0 .
$$

Self-preservation yields the power law decay

$$
K \sim t^{-1}
$$

with $L \sim t^{1 / 2}$ (cf. Speziale and Bernard 1992). Since, in this solution

$$
K L^{2} \sim \text { constant }
$$

then

$$
K L \dot{L} \sim t^{-1}
$$

It then follows that

$$
n=-1
$$

and, hence, $R_{i i}^{*} \sim k^{*-1}$ and $E^{*}\left(k^{*}\right) \sim k^{*}$ for $k^{*} \ll 1$ (by definition, $E^{*}\left(k^{*}\right)=2 \pi k^{* 2} R_{i i}^{*}$ ). The energy spectrum tensor is thus singular at $k^{*}=0$.

Based on the one-point fields, the governing equations for isotropic decay are:

$$
\begin{gathered}
\dot{K}=-\varepsilon \\
\dot{\varepsilon}=\frac{7}{3 \sqrt{15}} S_{K} R_{t}^{1 / 2} \frac{\varepsilon^{2}}{K}-\frac{7}{15} G \frac{\varepsilon^{2}}{K}
\end{gathered}
$$

where

$$
\begin{aligned}
& S_{K}=\frac{\overline{-\left(\frac{\partial u}{\partial x}\right)^{3}}}{\left[{ }_{\left.\left[\frac{\partial u}{\partial x}\right)^{2}\right]^{3 / 2}}^{3 / 2}\right.}=-\frac{6 \sqrt{15}}{7} \frac{\overline{\frac{\partial u_{i}}{\partial x_{k}} \frac{\partial u_{j}}{\partial x_{k}} \frac{\partial u_{i}}{\partial x_{j}}}}{\left[\frac{\partial u_{m} \frac{\partial u_{m}}{\partial x_{n}} \frac{\partial x_{n}}{\partial x_{n}}}{3 / 2}\right.} \\
& G=\frac{\overline{\frac{\partial^{2} u^{2} \partial^{2} u}{\partial x^{2}} \frac{\partial^{2}}{\partial x^{2}}} \cdot \overline{u^{2}}}{\left[{\overline{\left(\frac{\partial u}{\partial x}\right)^{2}}}^{2}\right.}=\frac{30}{7} \frac{\frac{\partial^{2} u_{i}}{\partial x_{j} \partial x_{k}} \frac{\partial^{2} u_{i}}{\partial x_{j} \partial x_{k}} K}{\left[\frac{\partial u_{m}}{\partial x_{n}} \frac{\partial u_{m}}{\partial x_{n}}\right]^{2}}
\end{aligned}
$$

are, respectively, the skewness and the palinstrophy coefficient. Self-preservation requires that

$$
S_{K}=\text { constant }, \quad G=\text { constant. }
$$

For $R_{t_{\infty}}>0$, this implies a $t^{-1}$ power law decay for large times as discussed earlier (see Speziale and Bernard 1992). 


\section{REFERENCES}

Bernard, P. S. and Speziale, C. G. 1992 "Bounded Energy States in Homogeneous Turbulent Shear Flow - An Alternative View," ASME J. Fluids Eng. 114, 29.

Clark, T. T. and Zemach, C. 1995 "A Spectral Model Applied to Homogeneous Turbulence," Phys. Fluids 7, 1674.

Speziale, C. G. and Bernard, P. S. 1992 "The Energy Decay in Self-Preserving Isotropic Turbulence Revisited," J. Fluid Mech. 241, 645. 


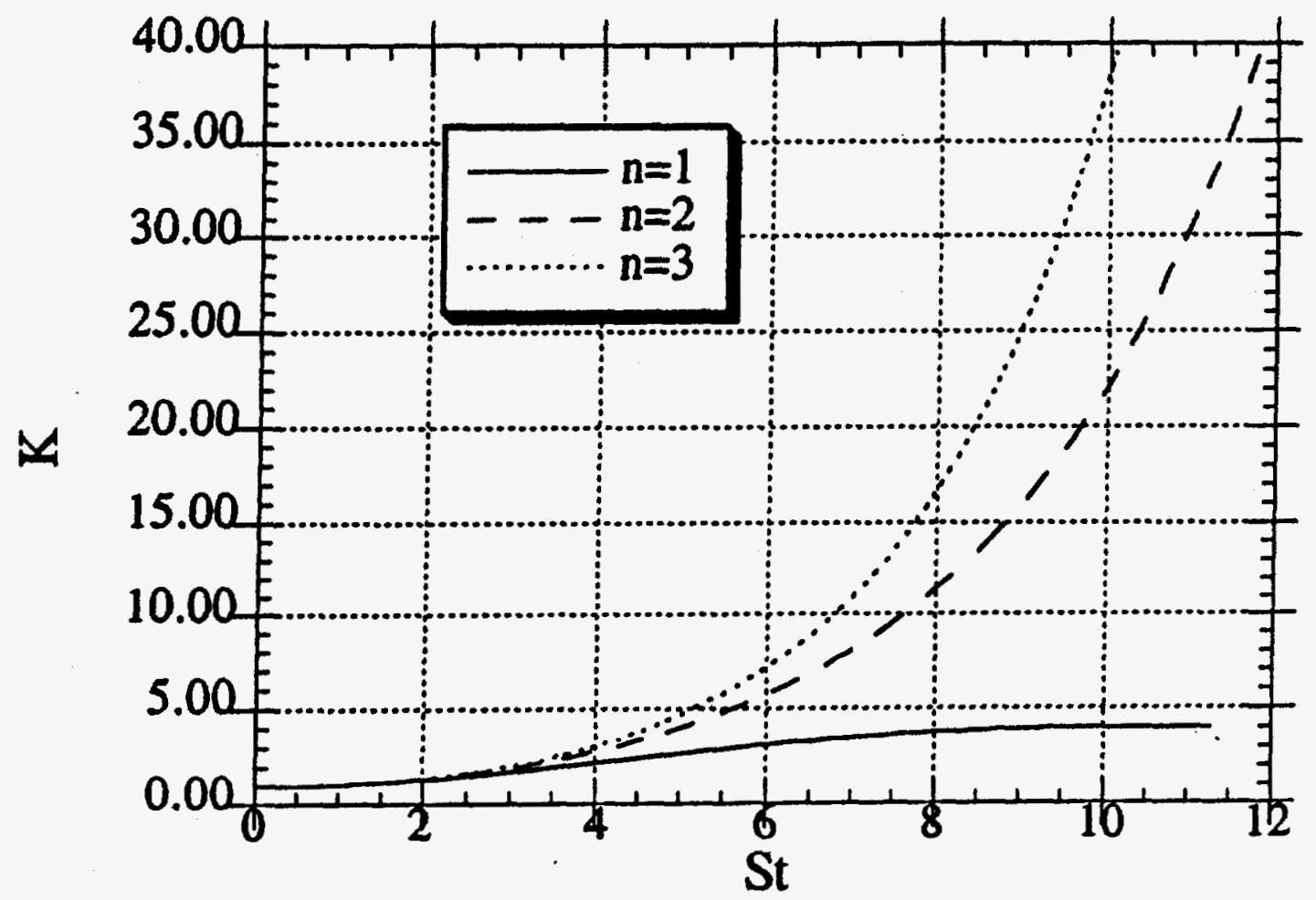

Figure 1. Time evolution of the turbulent kinetic energy in homogeneous shear flow predicted by the two-point closure of Clark and Zemach (1995). The initial energy spectrum, $E(k) \propto k^{n}$. 